\title{
Présentation de la copie nitrate de The Riddle of Rhodesia (Joseph Albrecht, 1933), un film sonore et teinté
}

Presentation of the nitrate copy of The Riddle of Rhodesia (Joseph Albrecht, 1933), a tinted sound film

\section{Mehdi Taïbi}

\section{OpenEdition}

Journals

Édition électronique

URL : https://journals.openedition.org/1895/4780

DOI : $10.4000 / 1895.4780$

ISSN : $1960-6176$

Éditeur

Association française de recherche sur l'histoire du cinéma (AFRHC)

Édition imprimée

Date de publication : 1 décembre 2013

Pagination : 145-148

ISBN : 978-2-37029-071-7

ISSN : 0769-0959

\section{Référence électronique}

Mehdi Taïbi, «Présentation de la copie nitrate de The Riddle of Rhodesia (Joseph Albrecht, 1933), un

film sonore et teinté », 1895. Mille huit cent quatre-vingt-quinze [En ligne], 71 | 2013, mis en ligne le 01 décembre 2016, consulté le 17 avril 2022. URL : http://journals.openedition.org/1895/4780 ; DOI :

https://doi.org/10.4000/1895.4780

\section{(c) AFRHC}




\section{Présentation de la copie nitrate de The Riddle of Rhodesia (Joseph Albrecht, 1933), un film sonore et teinté}

par Mehdi Taibi

The Riddle of Rhodesia; production: African Film Productions; réalisation: Joseph Albrecht; 1933 ; sonore; $35 \mathrm{~mm}$; teinté (rose et bleu); 223 mètres.

Ce film, proposé en complément du texte d'Anthony L'Abbate consacré à l'aventure des films sonores et teintés aux États-Unis, est disponible sur le DVD qui accompagne ce numéro. La copie provient des collections de la Cinémathèque française. Contrairement aux films tournés sur Sonochrome (film préteinté), The Riddle of Rhodesia a la particularité d'être un film sonore teinté après le développement du positif.

\section{Résumé du film}

Les ruines du Grand Zimbabwe semblent encore abriter d'impénétrables pans de mystères. Avec pour point de départ la découverte d'un site archéologique, le film dérive sur une rêverie qui tenterait d'expliquer la présence de ces énigmes de pierre. Par des couloirs étroits et d'épaisses murailles, on parcourt ces édifices monumentaux alors que les autochtones vivent à proximité dans de simples huttes. Afin d'illustrer le commentaire, des scènes du film King Solomon's Mines (Afrique du Sud, 1918) sont insérées. Plus loin, nous assistons à une reconstitution mettant en scène des nomades sémites dont les dromadaires sont utilisés dans le convoi de pierres précieuses. Quant aux indigènes, ils traversent le temps, inchangés. Relégués au rôle de figurants, ils plantent le décor, animent les vues et véhiculent un bon nombre des traits associés à l'iconographie du sauvage (archaïsme, tam-tam, sacrifice humain, etc.).

D’un point de vue chromatique, le film se divise en deux parties inégales, séparées par une collure enchainant un fondu au noir. La première partie, la plus longue, revêt un teintage rose, tandis que la seconde partie est plongée dans le sommeil paradoxal d'un teintage bleu. Les visions nocturnes, celles relatées au coin du feu par les contes et légendes des temps immémoriaux - la nuit des temps -, succèdent à un parcours diurne abordant les ruines que le commentaire va faire parler dans un récit partagé entre un éclairage faussement scientifique et des références confuses. Documentaire ou fiction? Mythe ou réalité? En définitive, c'est par le teintage que cette dualité empreinte d'incertitudes se trouve la mieux soulignée. 


\section{Le mythe entretenu du Grand Zimbabwe}

Plus de trois siècles après sa découverte par les Portugais au début du $\mathrm{XvI}^{\mathrm{e}}$ siècle le Grand Zimbabwe n'a cessé d'éveiller l'imaginaire des explorateurs et colonisateurs intrigués par son origine. En 1871, l'archéologue J. Theodore Bent (1952-1897), commandité par l'homme d'affaires et politicien Cecil Rhodes ${ }^{1}$ (1853-1902), établit un parallèle avec les Phéniciens, peuple alors en vogue dans le milieu archéologique naissant. À la même époque, l'explorateur allemand Karl Mauch (1837-1875) spécule sur une origine biblique en y localisant la cité d'Ophir de la Reine de Saba. De la civilisation Sumer à l'Atlantide, les théories fantasmatiques allaient perdurer, notamment à travers la littérature romanesque, alors que, dès 1905, des fouilles archéologiques avaient déjà prouvé l'origine africaine de la construction. En 1929, de nouveaux travaux scientifiques ont été menés par des archéologues britanniques qui ont confirmé la thèse d'une œuvre attribuée aux Shonas (population bantoue locale). Mais le film The Riddle of Rhodesia entretient le mystère comme l'annonce d'emblée le titre (riddle signifie énigme). Et, sans que le démenti avéré y soit clairement relayé, il est de nouveau question des mines du roi Salomon, de la Reine de Saba, des Phéniciens parmi les explications possibles. Justifiée par la rêverie, la version biblique est cette fois illustrée par une reconstitution où l'on voit, menée par des Himyarites, une caravane de dromadaires poursuivre ce cheminement de pensée plus onirique que scientifique. Quant aux huttes habitées par les tribus locales, elles suggèrent, comme un hiatus, qu'une filiation avec ce colossal et complexe édifice de pierres parait sans fondement. Le film préfere prétendre à une discutable neutralité en amalgamant preuves concrètes et imaginaire fabriqué. Le parti pris de l'énigme va jusqu'à dénigrer l'affirmation d'une ascendance indigène. Ou comment le fait scientifique est cité comme une hypothèse peu probante parmi d'autres hypothèses considérées comme plus prestigieuses.

Nous pouvons légitimement nous interroger sur la bonne foi de ces analyses qui dévalorisent volontairement les conclusions des études sérieuses connues depuis le début du $\mathrm{xx}^{\mathrm{e}}$ siècle. Les propositions fantaisistes sur lesquelles se focalise le film formulent davantage des tentatives de mystifications motivées ou encouragées par des enjeux stratégiques dans un contexte et un programme d'occupation coloniale durable. Admettre qu'il ait pu exister une civilisation noire capable de bâtir sur des bases solides reviendrait à remettre en question, d'une certaine manière, la mission civilisatrice de la colonisation entreprise dans le continent africain; quand bien même cette supposée mission pouvait n'être qu'un prétexte. C'est pourquoi convoquer les textes de l'Ancien Testament parait plus judicieux que de considérer l'évidence d'un lien séculaire entre les territoires et les populations que des nations européennes s’ingénient à coloniser. Reste à savoir si les faits historiques ont été niés dès le départ, ou tout simplement tenus comme inconcevables tant les idées reçues empreintes de théories racistes étaient ancrées dans une pensée dominante. C'est du côté de l'opportunisme que le dilemme s'est vite trouvé évacué. En présence de gisements de diamants et d'or dans la région, l’idée que ces sources

1. Figure emblématique de l'Empire britannique en Afrique, Cecil Rhodes fut l'administrateur du territoire qui portera son nom: la Rhodésie. 
de profit incommensurable étaient antérieurement exploitées par une "culture blanche» est plus que séduisante. Elle justifierait la reprise des activités et une réappropriation légitime des biens. Avec le diamant, l'enjeu est de taille...

Mais la version fantasmée se fissure lorsqu'elle se trouve confrontée à des preuves archéologiques qui échappent aux références culturelles des colonisateurs.

Le Grand Zimbabwe est le nom donné à ce site archéologique construit et occupé entre le $\mathrm{XI}^{\mathrm{e}}$ et le $\mathrm{XV}^{\mathrm{e}}$ siècle. On estime que l'enceinte abritait jusqu'à 18.000 personnes, sur une superficie de 722 hectares. Le Grand Zimbabwe est constitué de blocs de granit reliés par des murs formant des couloirs et de multiples enclos ou kraals. L'ensemble est dominé par une imposante acropole. Le système de kraals est une construction traditionnelle répandue dans les cultures d'Afrique australe, comme chez les Shonas et les Zoulous. Les spécialistes s'accordent à attribuer la réalisation du Grand Zimbabwe aux Gokomere qui sont les ancêtres des Shonas. Zimbabwe pourrait provenir de "Ziimba rembawe» qui signifie en ancienne langue shona "la Grande maison faite de pierres» ou encore, selon Bernard Lugan $^{2}$ "la cour du roi». La stratification de l'ensemble correspondait à une répartition sociale où les souverains résidaient au sommet alors que les murets des niveaux inférieurs servaient à enclore le bétail. La population qui résidait dans la cité du Grand Zimbabwe, capitale de l'empire Monomotapa, devait sa prospérité aux activités commerciales liées à l'extraction de l'or mais aussi à l'exploitation de l'ivoire et à l'élevage. Ces richesses étaient acheminées à plus de $500 \mathrm{kms}$ à l'est, dans le port de Sofala (dans l'actuel Mozambique), important comptoir où s'effectuaient des échanges avec la Perse ou la Chine, comme le prouvent "les fouilles [qui ont pu] mettre à jour un matériel archéologique composé de bijoux en or, d'objets en cuivre, ainsi que de grandes quantités de perles provenant d'ExtrêmeOrient et datées du $\mathrm{XIV}^{\mathrm{e}}$ siècle ${ }^{3}$. Parmi les raisons vraisemblables concernant l'abandon du site, Bernard Lugan évoque un seuil critique de concentration de population et un surpâturage provoquant l'épuisement des sols et la dégradation de l'environnement.

Jusqu'à l'indépendance de la Rhodésie du Sud devenue précisément Zimbabwe en 1980, les gouvernements successifs n'ont jamais reconnu le Grand Zimbabwe comme étant l'œuvre d'une société noire. Ses dirigeants veillaient même à censurer les publications qui n'allaient pas dans le sens de la version officielle qui privilégie l'énigme. Le monument est classé depuis 1986 dans la liste du patrimoine mondial de l'UNESCO.

\section{Un film sonore et teinté}

En 1933, année de la sortie du film The Riddle of Rhodesia, le cinéma sonore est en passe de devenir un procédé standard et exclusif. Néanmoins, la période demeure charnière et l'on peut encore

2. Bernard Lugan est maitre de Conférences à l'université de Lyon III, directeur-éditeur de l'Afrique réelle.

3. Bernard Lugan, "la Civilisation de Great Zimbabwe", juin 2000, texte disponible sur le site de Clio à l'adresse suivante : http://www.clio.fr/BIBLIOTHEQUE/la_civilisation_de_great_zimbabwe.asp [date de la dernière consultation : octobre 2013]. 
rencontrer une survivance du cinéma des trois décennies précédentes à travers certaines pratiques qu'avec le recul nous avons tendance à associer uniquement au cinéma muet. La copie nitrate de The Riddle of Rhodesia, conservée par la Cinémathèque française témoigne de cet entre-deux. La piste sonore à densité variable coexiste avec des images teintées. Lors du passage d'une teinte (rose) à l'autre (bleue) nous remarquons d'ailleurs une rupture d'intensité sonore manifeste se prolongeant sur tout le reste du film. En effet, avec le teintage, le film positif (image et support) était immergé dans un bain colorant, une teinture. Si bien que la piste optique obtenue par report n'était pas épargnée. Et la différence de contraste provoquée par les variations de teintes aura une incidence sur l'homogénéité du son. Par ce constat d'incompatibilité entre le teintage et la qualité du son nous pourrions hâtivement établir un lien de cause à effet expliquant l'abandon du teintage au moment de la globalisation du cinéma sonore. Ce serait ignorer que la firme Kodak avait conçu dès 1928 une pellicule préteintée ${ }^{4}$ appelée Sonochrome dont la vocation visait à pallier les dissonances dues aux interférences de la couleur sur le son. Avec le Sonochrome, les laboratoires Kodak proposaient jusqu'à dix-sept gammes de couleurs 5. La production de The Riddle of Rhodesia, en ayant fait l'économie du Sonochrome, s'était bornée à une pratique qui ne tenait pas compte des contraintes liées aux variations de contrastes qu'exige la piste optique.

La véritable raison de l'abandon des procédés tels que le teintage, le virage et les coloris au pochoir se trouve donc ailleurs et plus vraisemblablement dans la compétitivité émergente des procédés en couleurs dites naturelles et notamment ceux par synthèse soustractive. Les recherches ont ainsi privilégié ces procédés qui allaient bientôt connaitre un succès grandissant au détriment des teintures et coloris qui - ne l'oublions pas! - avaient d'abord été envisagés, dès le début de la photographie, comme des pis-aller.

En 2010, la Cinémathèque française a entrepris la restauration de The Riddle of Rhodesia. À partir de la copie teintée sur support nitrate un contretype noir et blanc a été tiré afin de recréer les teintes sur la nouvelle copie par le procédé Desmet. Parallèlement, le nouveau report optique a permis d'apporter un rééquilibrage du son cette fois incarné par une piste uniforme blanche sur fond cyan, standard actuel. Sur le positif nitrate, la densité variable suivait les "humeurs» de la teinte du rose - relativement neutre - au bleu qui atténue les exigences de contraste.

Par ailleurs, on notera sur les derniers mètres des bruits parasites dus aux traces d'humidité et de rouille qui affectent le signal sonore au point de rendre inaudibles les derniers mots du commentaire. L'image est aussi contaminée progressivement sur les dernières images de la copie nitrate. Ces défauts se sont trouvés reproduits sur le nouvel élément, par report pour le son, photographiés pour l'image.

4. Pathé avait déjà mis au point des films pré-teintés appelés «films à support teinté» dès la fin des années 1910. Il en est question dans l'édition de 1926 du manuel technique intitulé le Film vierge Pathé.

5. Voir infra l'article d'Anthony L'Abbate. 